\title{
A Splitting Iterative Method for Solving the Neutron Transport Equation
}

\author{
O. Awono ${ }^{1}$ and J. Tagoudjeu ${ }^{2}$ \\ ${ }^{1,2}$ Ecole Nationale Supérieure Polytechnique, University of Yaoundé I \\ ${ }^{2}$ Faculty of Science, University of Yaoundé I \\ ${ }^{1}$ Po.Box 8390 Yaoundé, Cameroon \\ ${ }^{2}$ Po.Box 812 Yaoundé, Cameroon \\ E-mail(corresp.): jtagoudjeu@gmail.com \\ E-mail: charles_awono@yahoo.fr
}

Received August 22, 2008; revised April 23, 2009; published online July 15, 2009

\begin{abstract}
This paper presents an iterative method based on a self-adjoint and $m$ accretive splitting for the numerical treatment of the steady state neutron transport equation. Theoretical analysis shows that this method converges unconditionally to the unique solution of the transport equation. The convergence of the method is numerically illustrated and compared with the standard Source Iteration method and multigrid method on sample problems in slab geometry and in two dimensional space.
\end{abstract}

Key words: Transport equation, self adjoint operator, m-accretive, operator splitting, iterative method.

\section{Introduction}

Iterative methods are intensively used for the solution of the transport equation $[1,2,6,9,10,11,14,15,17,18,19,20]$. The standard method is the source iteration method based on a decoupling between the differential and integral parts of the transport operator. Based on the natural splitting of the integral part of transport operator, methods such as Jacobi, Gauss-Seidel [18] and Successive over-relaxation (SOR) iteration have been successfully applied to transport problem. Using the same splitting, an adaptation to the infinite dimensional case of the minimal residual iteration method [1,2] has been proposed for the solution of the transport equation in slab geometry and in two dimensional space. This method has proved its efficiency and it competes with the SOR method.

In this work, focus is given on iterative methods for the numerical treatment of the single group steady state neutron transport equation in slab geometry and bounded convex domain of $\mathbb{R}^{n}(n=2,3)$. We introduce a two-step iteration method linked to a Self-adjoint and m-Accretive splitting of the transport 
operator. We investigate the convergence of this iterative method. Theoretical analysis shows that iterations converge unconditionally to the solution of the transport equation. An upper bound for the contraction factor of the iteration is derived. It is solely dependent on the spectrum of the Self-adjoint part of the transport operator. The convergence of the method is numerically illustrated and compared with the standard Source Iteration method, a spatial multigrid method and Krylov subspace methods such as Induced Dimension Reduction (IDR) [16], BiCGStab [7, 8] and a preconditioned GMRES methods [3] on sample problems in slab geometry and in two dimensional space.

The paper is organized as follows. In Section 2, we set the problem and present the splitting of the transport operator. Section 3 is devoted to the presentation and the convergence analysis of the new iterative method. Section 4 deals with an implementation of the method. The discretization and the numerical results obtained from example problems in slab geometry and two dimensional space are presented in Section 5. Some concluding remarks are given in Section 6.

\section{Mathematical Setting and Splitting Method}

\subsection{Basic definition}

Let us consider a Hilbert space $H$ with inner product $(\cdot, \cdot)_{H}$ and the associated norm $\|\cdot\|_{H}$. Let $X$ be an unbounded linear operator on $H$ with domain $\mathcal{D}(X)$. Let $I$ denotes the identity operator on $H$.

Definition 1. An operator $X: D(X) \subset H \rightarrow H$ is said to be $m$-accretive if $\forall u \in D(X),(X u, u)_{H} \geq 0$ and $\forall q \in H$, there exists $u \in D(X)$ such that $X u+u=q$.

We have the following results $[5,9]$ :

Theorem 1. Assume that $X$ is a m-accretive operator on $H$. Then:

1. $D(X)$ is dense in $H$.

2. The operator $X$ is closed.

3. $\forall \alpha>0,(I+\alpha X)$ is bijective from $D(X)$ to $H$, the operator $(I+\alpha X)^{-1}$ is bounded and $\left\|(I+\alpha X)^{-1}\right\|_{H} \leq 1$.

It follows from Theorem 1 that if $X$ is a $m$-accretive operator, then for any positive constant $\alpha$ it follows that the operator $(\alpha I+X)$ is positive definite and $\left\|(\alpha I+X)^{-1}\right\|_{H} \leq 1 / \alpha$. Thus $(\alpha I+X)^{-1}$ is bounded on $H$.

\subsection{Mathematical Setting}

The general single group, steady state first order neutron transport equation is given by:

$$
\Omega \cdot \nabla_{x} \psi(x, \Omega)+\sigma(x) \psi(x, \Omega)=\int_{S_{2}} \kappa\left(x, \Omega, \Omega^{\prime}\right) \psi(x, \Omega) d \Omega^{\prime}+q(x, \Omega),
$$


where $\sigma(x)$ is the total cross section; $\kappa\left(x, \Omega, \Omega^{\prime}\right)$ is a positive kernel specifying the scattering of particles; $q(x, \Omega)$ is a known particles source and $\psi(x, \Omega)$ represents the angular flux to be determined for all point $x=\left(x_{1}, x_{2}, x_{3}\right)$ in a bounded convex region $D \subset R^{3}$ with a sufficiently smooth boundary $\partial D$ and all possible travel directions $\Omega=(\theta, \varphi)$ on the unit sphere $S_{2}$. The boundary conditions prescribing the inflow of particles into the region $D$ reads:

$$
\psi(x, \Omega)=u_{0}(x, \Omega), \quad \forall(x, \Omega) \in \partial D_{-},
$$

where $\partial D_{-}=\left\{(x, \Omega) \in \partial D \times S_{2}, \Omega \cdot \vec{n}(x)<0\right\}, \vec{n}(x)$ being the outer unit normal to $D$ at point $x \in \partial D$. When $\frac{\partial \psi(x)}{\partial x_{3}}=0$, the problem (2.1)-(2.2) reduces to a $2 \mathrm{D}$-problem in space. In that case, the third component of the inner product $\Omega \cdot \nabla_{x} \psi$ is ignored.

In the case of slab geometry it is assumed that

$$
\frac{\partial \psi}{\partial x_{1}}=0, \quad \frac{\partial \psi}{\partial x_{2}}=0 .
$$

Defining $\mu=\cos (\theta)$, where $\theta$ denotes the angle between $\Omega$ and the $z$-axis, the angular flux becomes $\psi(x, \Omega) \equiv \psi(z, \mu)$ and problem $(2.1)-(2.2)$ reduces to $[9,11,13]$

$$
\left\{\begin{array}{l}
\mu \frac{\partial \psi}{\partial z}+\sigma(z) \psi-\int_{-1}^{1} \kappa\left(z, \mu, \mu^{\prime}\right) \psi\left(z, \mu^{\prime}\right) d \mu^{\prime}=q(z, \mu) \\
\psi\left(z_{l}, \mu\right)=g_{l}(\mu), \quad \text { for } \mu>0 \\
\psi\left(z_{r}, \mu\right)=g_{r}(\mu), \quad \text { for } \mu<0
\end{array}\right.
$$

In this case, we set $D=\left(z_{l}, z_{r}\right)$ and $S_{2}=[-1,1]$. Without a loss of generality, we assume in the following that vacuum boundary conditions are valid, i.e. $u_{0}(x, \Omega) \equiv 0$ in $(2.2)$ and $g_{l}(\mu) \equiv g_{r}(\mu) \equiv 0$ in $(2.3)$.

Let $Q=D \times S_{2}$. We define the spaces

$$
\begin{aligned}
& W^{2}(Q)=\left\{\psi \in L^{2}(Q), \quad \Omega \cdot \nabla_{x} \psi \in L^{2}(Q)\right\}, \\
& W_{0}=\left\{\psi \in W^{2}(Q), \psi(x, \Omega)=0, \quad \forall(x, \Omega) \in \partial D_{-}\right\} .
\end{aligned}
$$

The space $W^{2}(Q)$ is equipped with the norm

$$
\|\psi\|_{W^{2}}^{2}=\|\psi\|^{2}+\left\|\Omega \cdot \nabla_{x} \psi\right\|^{2}
$$

where $\|\cdot\|$ denotes the usual $L^{2}(Q)$ norm. The functions of $W^{2}(Q)$ have traces on $\partial D_{-}$in $L^{2}\left(\partial D \times S_{2}\right)[9]$. Let

$$
\left\{\begin{array}{l}
K \psi(x, \Omega)=\int_{S_{2}} \kappa\left(x, \Omega, \Omega^{\prime}\right) \psi\left(x, \Omega^{\prime}\right) d \Omega^{\prime} \\
A \psi(x, \Omega)=\Omega \cdot \nabla_{x} \psi(x, \Omega) \\
\Sigma \psi(x, \Omega)=\sigma(x) \psi(x, \Omega)
\end{array}\right.
$$


and $T=A+\Sigma-K$.

In the operator form, the equation (2.1) may be written as

$$
T \psi(x, \Omega)=q(x, \Omega) .
$$

We have $\mathcal{D}(K)=\mathcal{D}(\Sigma)=L^{2}(\Omega)$, where $\mathcal{D}(B)$ denotes the domain of operator $B$. It follows that $\mathcal{D}(T)=\mathcal{D}(A)=W_{0}$.

Proceeding as in $[1,2]$, we make the following assumptions:

$$
\begin{aligned}
& \sigma \in L^{\infty}(D), \exists \sigma_{0}>0 \text { such that } \sigma(x) \geq \sigma_{0} \text { a.e. on } D . \\
& \kappa\left(x, \Omega, \Omega^{\prime}\right)=\kappa\left(x, \Omega^{\prime}, \Omega\right) \text { and } \kappa \text { is positive. } \\
& \exists c \in[0,1), \int_{S_{2}} \kappa\left(x, \Omega, \Omega^{\prime}\right) d \Omega^{\prime} \leq \sigma_{0} c \text { a.e. on } Q .
\end{aligned}
$$

The above assumptions guarantee the following results $[2,9,13]$.

Theorem 2. The operators $T, K$ and $A$ verify the following properties:

1. The operator $T$ is positive definite.

2. The operators $K$ and $\Sigma-K$ are self-adjoint and positive definite.

3. The operator $A$ is $m$-accretive.

It then follows from Theorem 2 statement (1) that the solution of problem (2.1)-(2.2) exists and is unique in $W_{0}$, and from Theorem 2 statements (2), (3) that the operator $T$ admits a self adjoint and $m$-accretive splitting.

\subsection{The Splitting Methods}

The standard splitting of the transport operator consists of a decoupling between the differential and the integral parts as follows $-T=K-L$, where $L=A+\Sigma$. This splitting leads to the source iteration method: for a given $\psi^{0} \in \mathcal{D}(T)$, solve

$$
\left\{\begin{array}{l}
L \psi^{(n+1)}=K \psi^{n}+q \\
\psi^{(n+1)} \in \mathcal{D}(T) .
\end{array}\right.
$$

This method becomes extremely slow in the critical case. Several acceleration techniques of the convergence of the source iteration method such as Diffusion Synthetic Acceleration (DSA) [19] and multigrid algorithms have been introduced and studied [6, 10].

Let us consider now another natural splitting of the transport operator $T=(A+S)$, where $S=\Sigma-K$. Therefore for any positive constant $\alpha$, we have the following two-step splitting:

$$
\left\{\begin{array}{l}
T=(\alpha I+S)-(\alpha I-A) \\
T=(\alpha I+A)-(\alpha I-S) .
\end{array}\right.
$$




\section{Iterative Method}

We present in this section an iterative method for the solution of problem (2.1)(2.2). It relies on the splitting (2.4) and gives a two-step iteration algorithm.

\subsection{SA Splitting Iteration}

Let's take a constant $\alpha>0$. Given an initial guess $\psi^{(0)} \in D(T)$, for $k=0,1, \ldots$ until $\left\{\psi^{(k)}\right\}$ converges, calculate

$$
\left\{\begin{array}{l}
(\alpha I+S) \psi^{\left(k+\frac{1}{2}\right)}=(\alpha I-A) \psi^{(k)}+q, \\
(\alpha I+A) \psi^{(k+1)}=(\alpha I-S) \psi^{\left(k+\frac{1}{2}\right)}+q .
\end{array}\right.
$$

Remark 1. We have the following observations:

1. Since the operator $S$ is bounded, self-adjoint and positive definite, the operator $\alpha I+S$ is bounded, self-adjoint and positive definite for $\alpha>0$. Then it follows that $(\alpha I+S)^{-1}$ exists, it is bounded and self-adjoint. We also have that $\alpha I-S$ is a bounded self-adjoint operator.

2 . From the $m$-accretive property of the operator $A$, we deduce that for $\alpha>0$, the operator $\alpha I+A$ is invertible from $D(T)$ to $L^{2}(\Omega)$ and its inverse $(\alpha I+A)^{-1}$ is a bounded operator.

From equation (3.1), we deduce that $\psi^{(k+1)}$ satisfies

$$
(\alpha I+A) \psi^{(k+1)}=M(\alpha)(\alpha I+A) \psi^{(k)}+N(\alpha) q,
$$

where $M(\alpha)=S(\alpha) A(\alpha)$ and $N(\alpha)=2 \alpha(\alpha I+S)^{-1}$ with $S(\alpha)=(\alpha I-S)(\alpha I+$ $S)^{-1}$ and $A(\alpha)=(\alpha I-A)(\alpha I+A)^{-1}$. Therefore, the exact solution $\psi^{*}$ of the problem $(2.1)-(2.2)$ satisfies

$$
\left\|\psi^{(k+1)}-\psi^{*}\right\|_{(A+\alpha I)} \leq\|M(\alpha)\|\left\|\psi^{(k)}-\psi^{*}\right\|_{(A+\alpha I)},
$$

where $\|\psi\|_{(A+\alpha I)}=\|(\alpha I+A) \psi\|, \psi \in W_{0}$.

Theorem 3. Let $\alpha$ be a positive constant. The functional $\rho_{A(\alpha)}$ defined on $W_{0}$ by $\rho_{A(\alpha)}(u)=\|(\alpha I+A) u\|$ is a norm on $\mathcal{D}(T)$ equivalent to $\|\cdot\|_{W^{2}}$. The norm $\rho_{A(\alpha)}$ is denoted by $\|\cdot\|_{(A+\alpha I)}$.

Proof. Let $\alpha$ be a positive constant. It can be easily seen from the linearity of the operator $(\alpha I+A)$ and the properties of the norm $\|\cdot\|$ that $\forall u, v \in W_{0}$ and any constant $\beta$ we have

$$
\rho_{A(\alpha)}(u+v) \leq \rho_{A(\alpha)}(u)+\rho_{A(\alpha)}(v) \text { and } \rho_{A(\alpha)}(\beta u)=|\beta| \rho_{A(\alpha)}(u) .
$$

Moreover, since $A$ is $m$-accretive, $\alpha I+A$ is positive definite and $\rho_{A(\alpha)}(u)=0$ if and and only if $u=0$. Then it follows that $\rho_{A(\alpha)}$ is a norm on $\mathcal{D}(T)$.

Let $u \in W_{0}$. We have

$$
\left(\rho_{A(\alpha)}(u)\right)^{2}=\alpha^{2}\|u\|^{2}+\|A u\|^{2}+2 \alpha(u, A u) .
$$


Since $(A u, u) \geq 0(A$ is $m$-accretive $)$ and $(A u, u) \leq \frac{\|u\|^{2}+\|A u\|^{2}}{2}$, we have

$$
\min \left\{\alpha^{2}, 1\right\}\|u\|_{W^{2}}^{2} \leq\left(\rho_{A(\alpha)}(u)\right)^{2} \leq(\alpha+1)^{2}\|u\|_{W^{2}}^{2} .
$$

It follows that, the norms $\|\cdot\|_{W^{2}}$ and $\rho_{A(\alpha)}$ are equivalent in $W_{0}$.

Since $\|\cdot\|_{(A+\alpha I)}$ is a norm in $W_{0}$, it is well known that the iteration method (3.1) converges (in the sense of the norm $\|\cdot\|_{(A+\alpha I)}$ ) if the operator $M(\alpha)$ satisfies $\|M(\alpha)\|<1$. We have the following Lemma.

Lemma 1. Let $\alpha$ be a positive constant. If the operator $X$ is $m$-accretive in a Hilbert space $H$ with the inner product $(\cdot, \cdot)_{H}$ and the associate norm $\|\cdot\|_{H}$, then the operator $X(\alpha)$ defined by

$$
X(\alpha)=(\alpha I-X)(\alpha I+X)^{-1}
$$

satisfies $\|X(\alpha)\|_{H} \leq 1$.

Proof. Let $\alpha>0$. If $X$ is $m$-accretive, then $(\alpha I+X)$ is bijective from $D(X)$ to $H$. The operator $(\alpha I+X)^{-1}$ is bounded and, for $\psi \in D(X),(X \psi, \psi)_{H} \geq 0$. Moreover, we have

$$
\|(\alpha I-X) \psi\|_{H}^{2}-\|(\alpha I+X) \psi\|_{H}^{2}=-4 \alpha(X \psi, \psi)_{H} \leq 0 .
$$

Taking $\psi=(\alpha I+X)^{-1} \varphi,(\varphi \in H)$, we obtain

$$
\left\|(\alpha I-X)(\alpha I+X)^{-1} \varphi\right\|_{H}^{2}=\|X(\alpha) \varphi\|_{H}^{2} \leq\|\varphi\|_{H}^{2} .
$$

It follows that $\|X(\alpha)\| \leq 1$.

Theorem 4. [Convergence of the SAS iteration method.] Let $\alpha$ be a positive constant. The norm $\|M(\alpha)\|$ of the operator $M(\alpha)$ is bounded by

$$
\beta(\alpha)=\sup _{\lambda \in \sigma(S)}\left|\frac{\alpha-\lambda}{\alpha+\lambda}\right|,
$$

where $\sigma(S)$ is the spectrum of the operator $S$. Therefore it holds that

$$
\|(M(\alpha) \| \leq \beta(\alpha)<1, \quad \forall \alpha>0
$$

and the SAS iteration converges to the unique solution $\psi^{*} \in \mathcal{D}(T)$ of the problem (2.1)-(2.2). The optimal parameter $\bar{\alpha}$ which minimizes the bound $\beta(\alpha)$ is given by (see, [12]) $\bar{\alpha}=\sqrt{\lambda_{\min } \lambda_{\max }}$ and

$$
\beta(\bar{\alpha})=\left(\sqrt{\lambda_{\max }}-\sqrt{\lambda_{\min }}\right) /\left(\sqrt{\lambda_{\max }}+\sqrt{\lambda_{\min }}\right),
$$

where $\lambda_{\min }$ and $\lambda_{\max }$ denote respectively the lower and the upper bounds of the spectrum of the operator $S$. 
Proof. For $\alpha>0, A(\alpha)$ and $S(\alpha)$ are bounded operators from $L^{2}(Q)$ to $\mathcal{D}(T)$ and $L^{2}(Q)$ respectively. We have

$$
\|M(\alpha)\| \leq\|S(\alpha)\|\|A(\alpha)\|
$$

Since $S$ is a bounded, self-adjoint operator in $L^{2}(Q)$, we have

$$
\|S(\alpha)\|=\left\|(\alpha I-S)(\alpha I+S)^{-1}\right\|=\sup _{\lambda \in \sigma(S)}\left|\frac{\alpha-\lambda}{\alpha+\lambda}\right|=\beta(\alpha) .
$$

It holds from the positivity of $\alpha$ and $\lambda$ that $\beta(\alpha)<1$. The proof of the theorem is achieved if the norm of operator $A(\alpha)$ verifies $\|A(\alpha)\| \leq 1$. The operator $A$ is $m$-accretive in the Hilbert space $L^{2}(Q)$. It follows from Lemma 1 that $\|A(\alpha)\| \leq 1$. Therefore, $\|M(\alpha)\| \leq \beta(\alpha)<1$.

From the equivalence between the norms $\|\cdot\|_{W^{2}}$ and $\|\cdot\|_{(A+\alpha I)}$ we get the convergence with respect to the norm $\|\cdot\|_{W^{2}}$ in $\mathcal{D}(T)$. Additionally, since

$$
\left\|u^{(k+1)}-u^{*}\right\| \leq\left\|u^{(k+1)}-u^{*}\right\|_{W^{2}}, \quad k=0,1,2, \cdots,
$$

we have

$$
\lim _{k \rightarrow+\infty}\left\|u^{(k)}-u^{*}\right\|=\lim _{k \rightarrow+\infty}\left\|u^{(k)}-u^{*}\right\|_{W^{2}}=0 .
$$

Thus the sequence $u^{(k)}$ converges in $\mathcal{D}(T)$ with respect to the norm $\|\cdot\|$.

The results of Theorem 4 show that $\beta(\alpha)$ is an upper bound of the contraction factor of the SAS iteration in the sense of the norm $\|\cdot\|_{(A+\alpha I)}$ in $\mathcal{D}(T)$. It follows from assumption (A1), (A2) and (A3) that $[9,13]:\|K\| \leq \sigma_{0} c$ and

$$
(S u, u) \geq \sigma_{0}(1-c)\|u\|^{2}, \quad \forall u \in L^{2}(Q) .
$$

Since $S$ is self-adjoint, bounded and positive definite, $S^{-1}$ is self-adjoint, bounded and positive definite on the Hilbert space $L^{2}(Q)$. It holds that [4]:

$$
\frac{1}{\lambda_{\max }} \leq\left\|S^{-1}\right\|=\sup _{\|u\| \neq 0} \frac{\|u\|}{\|S u\|} \leq \frac{1}{\sigma_{0}(1-c)} .
$$

It follows that $\lambda_{\min } \geq \sigma_{0}(1-c)$ and

$$
\sigma(S) \subset\left[\sigma_{0}(1-c), \sigma_{1}+\sigma_{0} c\right]
$$

where $\sigma_{1}=\sup _{x \in D}|\sigma(x)|$. We then have

$$
\beta(\alpha) \leq \sup _{\lambda \in\left[\sigma_{0}(1-c), \sigma_{1}+\sigma_{0} c\right]}\left|\frac{\lambda-\alpha}{\lambda+\alpha}\right|<1 .
$$

Each step of the SAS iterative method is constituted of two-half steps which require finding solutions of linear equations with operators $(\alpha I+S)$ and $(\alpha I+$ $A)$. Exact solutions of these equations are generally not available. These linear equations can be solved approximately using appropriate methods with respect to the properties of each operators. This results in the following inexact Self-adjoint and $m$-Accretive splitting (ISAS) iteration for solving the linear equation (2.1)-(2.2). 


\subsection{ISAS Iteration Method}

Given an initial guess $\bar{\psi}^{(0)} \in \mathcal{D}(T)$. For $k=0,1,2, \ldots$ until $\left\{\bar{\psi}^{(k)}\right\}$ converges, solve $\bar{\psi}^{\left(k+\frac{1}{2}\right)}$ approximately from

$$
(\alpha I+S) \bar{\psi}^{\left(k+\frac{1}{2}\right)} \approx(\alpha I-A) \bar{\psi}^{(k)}+q
$$

by employing an inner iteration (e.g the Conjugate Gradient method) with $\bar{\psi}^{(k)}$ as the initial guess, then solve $\bar{\psi}^{(k+1)}$ approximately from

$$
(\alpha I+A) \bar{\psi}^{(k+1)} \approx(\alpha I-S) \bar{\psi}^{\left(k+\frac{1}{2}\right)}+q,
$$

where $\alpha$ is a given positive constant. We now set

$$
M_{1}=\alpha I+S, \quad M_{2}=\alpha I+A, \quad N_{1}=\alpha I-A, \quad N_{2}=\alpha I-S .
$$

Theorem 5. [Convergence of the ISAS iteration method.] If the iterative sequence $\left\{\bar{\psi}^{(k)}\right\}$ is defined as follows

$$
\bar{\psi}^{\left(k+\frac{1}{2}\right)}=\bar{\psi}^{(k)}+\bar{\varphi}^{(k)} \text {, with } M_{1} \bar{\varphi}^{(k)}=\bar{\phi}^{(k)}+\bar{p}^{(k)},
$$

satisfying $\left\|\bar{p}^{(k)}\right\| \leq \epsilon_{k}\left\|\bar{\phi}^{(k)}\right\|$, where $\bar{\phi}^{(k)}=q-T \bar{\psi}^{(k)}$, and

$$
\bar{\psi}^{(k+1)}=\bar{\psi}^{\left(k+\frac{1}{2}\right)}+\bar{\varphi}^{\left(k+\frac{1}{2}\right)} \text {, with } M_{2} \bar{\varphi}^{\left(k+\frac{1}{2}\right)}=\bar{\phi}^{\left(k+\frac{1}{2}\right)}+\bar{\tau}^{\left(k+\frac{1}{2}\right)},
$$

satisfying $\left\|\bar{\tau}^{\left(k+\frac{1}{2}\right)}\right\| \leq \eta_{k}\left\|\bar{\phi}^{(k)}\right\|$, where $\bar{\phi}^{\left(k+\frac{1}{2}\right)}=q-T \bar{\psi}^{\left(k+\frac{1}{2}\right)}$, then $\left\{\bar{\psi}^{(k)}\right\}$ is given of the form

$$
\begin{aligned}
\bar{\psi}^{(k+1)}=M_{2}^{-1} N_{2} M_{1}^{-1} N_{1} \bar{\psi}^{(k)} & +M_{2}^{-1}\left(I+N_{2} M_{1}^{-1}\right) q \\
& +M_{2}^{-1}\left(N_{2} M_{1}^{-1} \bar{p}^{(k)}+\bar{\tau}^{(k+1 / 2)}\right) .
\end{aligned}
$$

Moreover, if $\psi^{*} \in \mathcal{D}(T)$ is the exact solution of the equation (2.1)-(2.2), then we have for $k=0,1,2, \ldots$ :

$$
\left\|M_{2}\left(\bar{\psi}^{(k+1)}-\psi^{*}\right)\right\| \leq\left(\beta+\mu \theta\left(\mu \epsilon_{k}+\eta_{k}\right)\right)\left\|M_{2}\left(\bar{\psi}^{(k)}-\psi^{*}\right)\right\|,
$$

where

$$
\beta=\left\|N_{2} M_{1}^{-1} N_{1} M_{2}^{-1}\right\| \leq \beta(\alpha), \quad \mu=\left\|N_{2} M_{1}^{-1}\right\|, \quad \theta=\left\|T M_{2}^{-1}\right\| .
$$

In particular, if $\beta(\alpha)+\theta\left(\mu \epsilon_{\max }+\eta_{\max }\right)<1$, then the iterative sequence $\left\{\bar{\psi}^{(k)}\right\}$ converges to $\psi^{*} \in D(T)$, where $\epsilon_{\max }=\max _{k}\left\{\epsilon_{k}\right\}$ and $\eta_{\max }=\max _{k}\left\{\eta_{k}\right\}$.

Proof. From (3.2), we have

$$
\begin{aligned}
\bar{\psi}^{\left(k+\frac{1}{2}\right)} & =\bar{\psi}^{(k)}+M_{1}^{-1}\left(\bar{\phi}^{(k)}+\bar{p}^{(k)}\right)=\left(I-M_{1}^{-1} T\right) \bar{\psi}^{(k)}+M_{1}^{-1} q+M_{1}^{-1} \bar{p}^{(k)} \\
& =M_{1}^{-1} N_{1} \bar{\psi}^{(k)}+M_{1}^{-1} q+M_{1}^{-1} \bar{p}^{(k)} .
\end{aligned}
$$


Similarly, from (3.3) we have

$$
\begin{aligned}
\bar{\psi}^{(k+1)} & =M_{2}^{-1} N_{2} \bar{\psi}^{\left(k+\frac{1}{2}\right)}+M_{2}^{-1} q+M_{2}^{-1} \bar{\tau}^{\left(k+\frac{1}{2}\right)} \\
& =M_{2}^{-1} N_{2}\left(M_{1}^{-1} N_{1} \bar{\psi}^{(k)}+M_{1}^{-1} q+M_{1}^{-1} \bar{p}^{(k)}\right)+M_{2}^{-1} q+M_{2}^{-1} \bar{\tau}^{\left(k+\frac{1}{2}\right)} \\
& =M_{2}^{-1} N_{2} M_{1}^{-1} N_{1} \bar{\psi}^{(k)}+M_{2}^{-1}\left(N_{2} M_{1}^{-1}+I\right) q+M_{2}^{-1}\left(N_{2} M_{1}^{-1} \bar{p}^{(k)}+\bar{\tau}^{\left(k+\frac{1}{2}\right)}\right) .
\end{aligned}
$$

The exact solution $\psi^{*}$ of equation (2.1)-(2.2) satisfies

$$
\psi^{*}=M_{2}^{-1} N_{2} M_{1}^{-1} N_{1} \psi^{*}+M_{2}^{-1}\left(N_{2} M_{1}^{-1}+I\right) q .
$$

By subtracting (3.5) from (3.4), we have

$$
\bar{\psi}^{(k+1)}-\psi^{*}=M_{2}^{-1} N_{2} M_{1}^{-1} N_{1}\left(\bar{\psi}^{(k)}-\psi^{*}\right)+M_{2}^{-1}\left(N_{2} M_{1}^{-1} \bar{p}^{(k)}+\bar{\tau}^{\left(k+\frac{1}{2}\right)}\right) .
$$

Multiplying (3.6) by $M_{2}$ and taking norms on both sides, we obtain

$$
\begin{aligned}
& \left\|M_{2}\left(\bar{\psi}^{(k+1)}-\psi^{*}\right)\right\| \\
& \quad \leq\left\|N_{2} M_{1}^{-1} N_{1} M_{2}^{-1} M_{2}\left(\bar{\psi}^{(k)}-\psi^{*}\right)\right\|+\left\|N_{2} M_{1}^{-1}\right\|\left\|\bar{p}^{(k)}\right\|+\left\|\bar{\tau}^{(k+1 / 2)}\right\| \\
& \quad \leq\left\|N_{2} M_{1}^{-1} N_{1} M_{2}^{-1}\right\|\left\|M_{2}\left(\bar{\psi}^{(k)}-\psi^{*}\right)\right\|+\left\|N_{2} M_{1}^{-1}\right\|\left\|\bar{p}^{(k)}\right\|+\left\|\bar{\tau}^{(k+1 / 2)}\right\| .
\end{aligned}
$$

Noticing that

$$
\left\|\bar{\phi}^{(k)}\right\|=\left\|b-T \bar{\psi}^{(k)}\right\|=\left\|T\left(\psi^{*}-\bar{\psi}^{(k)}\right)\right\| \leq\left\|T M_{2}^{-1}\right\|\left\|M_{2}\left(\psi^{*}-\bar{\psi}^{(k)}\right)\right\|,
$$

by the definition of the sequences $\left\{\bar{p}^{(k)}\right\}$ and $\left\{\tau^{\left(k+\frac{1}{2}\right)}\right\}$ we have

$$
\begin{aligned}
& \left\|\bar{p}^{(k)}\right\| \leq \epsilon_{k}\left\|\bar{\phi}^{(k)}\right\| \leq \epsilon_{k}\left\|T M_{2}^{-1}\right\|\left\|M_{2}\left(\psi^{*}-\bar{\psi}^{(k)}\right)\right\|, \\
& \left\|\bar{\tau}^{\left(k+\frac{1}{2}\right)}\right\| \leq \eta_{k}\left\|\bar{\phi}^{(k}\right\| \leq \eta_{k}\left\|T M_{2}^{-1}\right\|\left\|M_{2}\left(\psi^{*}-\bar{\psi}^{(k)}\right)\right\| .
\end{aligned}
$$

Through substitution, we finally obtain

$$
\begin{aligned}
& \left\|M_{2}\left(\bar{\psi}^{(k+1)}-\psi^{*}\right)\right\| \leq\left\|N_{2} M_{1}^{-1} N_{1} M_{2}^{-1}\right\|\left\|M_{2}\left(\bar{\psi}^{(k)}-\psi^{*}\right)\right\| \\
& \quad+\epsilon_{k}\left\|N_{2} M_{1}^{-1}\right\|\left\|T M_{2}^{-1}\right\|\left\|M_{2}\left(\psi^{*}-\bar{\psi}^{(k)}\right)\right\|+\eta_{k}\left\|T M_{2}^{-1}\right\|\left\|M_{2}\left(\bar{\psi}^{(k)}-\bar{\psi}^{*}\right)\right\| \\
& \quad \leq\left(\beta+\theta\left(\mu \epsilon_{k}+\eta_{k}\right)\right)\left\|M_{2}\left(\bar{\psi}^{(k)}-\psi^{*}\right)\right\| .
\end{aligned}
$$

Remark 2. If the first (resp., the second) inner equation of (3.1) can be solved exactly, then the sequence $\epsilon_{k}$ (resp., $\eta_{k}$ ) is equal to zero and the convergence rate of the ISAS iteration is given by $R=\beta(\alpha)+\theta \eta_{\max }$ (resp., $R=\beta(\alpha)+$ $\left.\mu \theta \epsilon_{\max }\right)$. It then follows that the convergence rate of the ISAS iterations is reduced to the one of SAS iteration when the two inner equations of (3.1) are exactly solved.

Theorem 6. In the case of isotropic scattering where the integral operator is defined by

$$
K \psi=\sigma(x) c P \psi
$$


with

$$
P \psi=\frac{1}{\left|S_{2}\right|} \int_{S_{2}} \psi\left(x, \Omega^{\prime}\right) d \Omega^{\prime}, \quad\left|S_{2}\right|=\int_{S_{2}} d \Omega^{\prime} ;
$$

the inverse of the operator $(\alpha I+S)$ is given by:

$$
(\alpha I+S)^{-1}=\frac{1}{\sigma(x)(1-c)+\alpha} P+\frac{1}{\sigma(x)+\alpha}(I-P) .
$$

Proof. The operator $(\alpha I+S)$ can be written as $(\alpha I+S)=\lambda(P+\epsilon(I-P))$, where $\lambda=\sigma(x)(1-c)+\alpha$ and $\epsilon=(\sigma(x)+\alpha) / \lambda$. Since $P^{2}=P$, one has

$$
(P+\epsilon(I-P))\left(P+\frac{1}{\epsilon}(I-P)\right)=I .
$$

It then follows that $(\alpha I+S)^{-1}=(P+(I-P) / \epsilon) / \lambda$.

Therefore, in the case of isotropic scattering, the first subproblem of the system (3.1) can be solved explicitly. Moreover, the two-step iteration method defined by (3.1) can be reduced to the following iteration: for a given $\psi^{(0)} \in W_{0}$, compute

$$
(\alpha I+A) \psi^{(k+1)}=B\left[(\alpha I-A) \psi^{(k)}+q\right]+q, \text { for } k=0,1, \ldots,
$$

where

$$
B=\left(\frac{\alpha-\sigma(1-c)}{\alpha+\sigma(1-c)}-\frac{\alpha-\sigma}{\alpha+\sigma}\right) P+\frac{\alpha-\sigma}{\alpha+\sigma} I .
$$

\section{An Implementation of the ISAS Method}

The SAS iteration (3.1), can be written as : Given an initial guess $\varphi^{(0)} \in L^{2}(\Omega)$, for $k=0,1, \ldots$ until $\left\{\varphi^{(k)}\right\}$ converges, calculate

$$
\left\{\begin{array}{l}
(\alpha I+S) \varphi^{\left(k+\frac{1}{2}\right)}=(\alpha I-A)(\alpha I+A)^{-1} \varphi^{(k)}+q \\
\varphi^{(k+1)}=(\alpha I-S) \varphi^{\left(k+\frac{1}{2}\right)}+q
\end{array}\right.
$$

where

$$
\varphi^{(k)}=(\alpha I+A) \psi^{(k)}, \quad \varphi^{\left(k+\frac{1}{2}\right)}=\psi^{\left(k+\frac{1}{2}\right)} .
$$

At each step $k$ of the iteration method (4.1), we have to solve a linear equation

$$
\mathcal{A}(\alpha) F=q(\alpha)
$$

where $\mathcal{A}(\alpha)=(\alpha I+S)$ and $q(\alpha)=(\alpha I-A)(\alpha I+A)^{-1} \varphi^{(k)}+q$. The solution of equation (4.2) is then used to compute $\varphi^{(k+1)}$. An infinite dimensional adaptation of the Conjugate Gradient method is employed to solve the equation (4.2). We have the following algorithm for the ISAS method:

Let $\psi^{(0)} \in \mathcal{D}(T), \mathcal{R}^{(0)}=q-T \psi^{(0)}, q(\alpha)=(\alpha I-A) \psi^{(0)}+q$. 
While $\left\|\mathcal{R}^{(k)}\right\|>\epsilon$ do

begin

end

$$
\begin{aligned}
& \text { solve } \mathcal{A}(\alpha) F=q(\alpha) \text { by } \mathrm{CG} \text { method; } \\
& \text { compute } \varphi^{(k+1)}=(\alpha I-S) F+q ; \\
& \text { compute } q(\alpha)=(\alpha I-A)(\alpha I+A)^{-1} \varphi^{(k+1)} \\
& \text { compute } \mathcal{R}^{(k+1)}=q-\left(I-(\alpha I-S)(\alpha I+A)^{-1}\right) \varphi^{(k+1)}
\end{aligned}
$$

In the previous algorithms, we have to make clear how the right hand side $q(\alpha)$ is computed, since it contains the inverse operator $(\alpha I+A)^{-1}$. Let $\varphi \in$ $L^{2}(Q)$, we describe in the following how to compute $\phi=(\alpha I-A)(\alpha I+A)^{-1} \varphi$. We have

$$
\phi=(\alpha I-A) f
$$

where $f$ verifies the linear equation

$$
(\alpha I+A) f=\varphi, \quad f \in \mathcal{D}(T) .
$$

Once $f$ is calculated, the product (4.3) can be easily computed. The differential equation (4.4) can be solved numerically. The discrete solution of (4.4) can be approximated by preconditioned Krylov methods such as the BiCGStab algorithm with Gauss-Seidel preconditioner which was applied and investigated for simulation of convection dominated flows and heat conduction problems with nonlinear convection flows through boundaries of the domain $[7,8]$. In the case of one and two dimensional spaces, where DSN schemes are used, the direct sweeping algorithm [9] is advised since it solve the problem in $O(N)$ operations, $N$ being the size of the problem.

\section{Discretization and Numerical Results}

Here, we investigate the numerical simulations for one dimensional slab geometry and two dimensional case.

\subsection{One Dimensional Case}

For the discretization of the problem (4.4), we introduce a finite set of $J$ discrete angular directions $\left\{\mu_{k}\right\}_{l=1}^{J} \in[-1,1]$, which are nonzero and symmetric about the origin. Using the discrete directions $\mu_{l}(l=1,2, \ldots, J)$, the semi-discrete formulation of the problem (4.4) can be stated as follows:

$$
\left\{\begin{array}{l}
\frac{\mu_{l} \partial f_{l}}{\partial x}+\alpha f_{l}=\varphi_{k}, \text { in }(a, b), \\
f\left(a, \mu_{l}\right)=f\left(b,-\mu_{l}\right)=0, \quad \mu_{l}>0
\end{array}\right.
$$

where $f_{l}(x)=f\left(x, \mu_{l}\right), l=1,2, \ldots, J$.

The fully discrete form of the problem (4.4) is obtained by discretizing the equation (5.1). The numerical grid of the spatial domain is defined by: $D_{h}=\left\{x_{i}, 0 \leq i \leq N\right\}$, where $x_{0}=a, x_{i}=x_{i-1}+(\Delta x)_{i}(0<i<N), x_{N}=b$ and $h=\max _{0<i \leq N}(\Delta x)_{i}$. The cell center grid points are $x_{i+\frac{1}{2}}=\left(x_{i+1}+x_{i}\right) / 2$ and $(\Delta x)_{i+\frac{1}{2}}=x_{i+1}-x_{i}=h_{i}$. 
Using the difference method based on the control volume approach and cell averaging, a fully discrete form of the transport problem (4.4) can be written as follows:

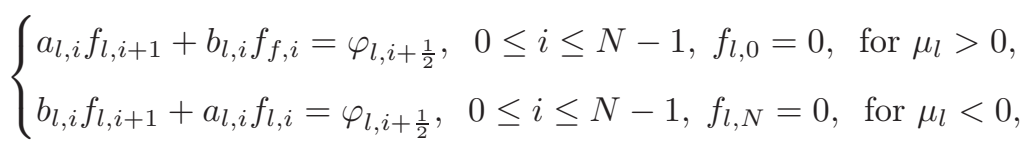

where $a_{l, i}=\left|\mu_{l}\right| / h_{i}+\alpha / 2$ and $b_{l, i}=-\left|\mu_{l}\right| / h_{i}+\alpha / 2$. The matrices of the discrete systems (5.2) are sparse. These systems are solved by using the direct forward substitution for $\mu_{l}>0$ and the backward substitution for $\mu_{l}<0$, since their matrices are of triangular form.

\subsection{Two Dimensional Case}

For the solution of (4.4) we introduce a finite set of $J$ discrete angular directions $\Omega_{J}=\left\{\omega_{i}=\left(\mu_{i}, \eta_{i}, \xi_{i}\right)\right\}_{i=1}^{i=J} \subset S^{1}$, which are nonzero and symmetric about the origin. Using the discrete directions of the set $\Omega_{J}$, the semi-discrete formulation of the problem (4.4) reads:

$$
\begin{cases}\mu_{l} \frac{\partial f_{l}}{\partial x}+\eta_{l} \frac{\partial f_{l}}{\partial y}+\alpha f_{l}=\varphi_{l}, & \text { in } D \times \Omega_{J} \\ f_{l}(\mathbf{x})=0, & \text { in } \partial D_{-} \times \Omega_{J}\end{cases}
$$

where we denote $f_{l}(x, y)=f\left(x, y, \mu_{l}, \eta_{l}\right)(1 \leq l \leq J)$.

We assume that $D$ is the rectangular domain defined by $D=(a, b) \times(c, d)$. The numerical grid is defined by:

$$
D_{h}=\left\{\left(x_{i}, y_{j}\right), \quad 0 \leq i \leq N, \quad 0 \leq i \leq M\right\}
$$

where $x_{0}=a, x_{i}=x_{i-1}+(\Delta x)_{i}, x_{N}=b, y_{0}=c, y_{j}=y_{j-1}+(\Delta y)_{j}$, $y_{M}=d$ and $h=\max _{i j}\left((\Delta x)_{i},(\Delta y)_{j}\right)$. The cell center grid points are defined as: $x_{i+\frac{1}{2}}=\left(x_{i+1}-x_{i}\right) / 2, y_{j+\frac{1}{2}}=\left(y_{j+1}-y_{j}\right) / 2,(\Delta x)_{i+\frac{1}{2}}=x_{i+1}-x_{i}$ and $(\Delta y)_{j+\frac{1}{2}}=y_{j+1}-y_{j}$. Denoting by $f_{i j}$ the approximation of the function $f$ at the node $\left(x_{i}, y_{j}\right)$, the value of $f$ at the cell center is approximated by:

$$
f_{i+\frac{1}{2} j+\frac{1}{2}}=\frac{f_{i j}+f_{i+1 j}+f_{i j+1}+f_{i+1 j+1}}{4} .
$$

Using the centered difference method for the approximation of derivative, a fully discrete form of problem (4.4) reads :

$$
\mu_{l} \frac{f_{l, i+1 j+\frac{1}{2}}-f_{l, i j+\frac{1}{2}}}{(\Delta x)_{i+\frac{1}{2}}}+\eta_{l} \frac{f_{l, i+\frac{1}{2} j+1}-f_{l, i+\frac{1}{2} j}}{(\Delta y)_{i+\frac{1}{2}}}+\alpha f_{l, i+\frac{1}{2} j+\frac{1}{2}}=\varphi_{l, i+\frac{1}{2} j+\frac{1}{2}} .
$$

Using the relations $f_{i j+\frac{1}{2}}=\left(f_{i j}+f_{i j+1}\right) / 2$ and $f_{i+\frac{1}{2} j}=\left(f_{i j}+f_{i+1 j}\right) / 2$, system (5.3) can be written as:

$$
a_{l, i j} f_{l, i j}+b_{l, i j} f_{l, i j+1}+c_{l, i j} f_{l, i+1 j}+d_{l, i j} f_{l, i+1 j+1}=\varphi_{l, i+\frac{1}{2} j+\frac{1}{2}},
$$

where $a_{l, i j}=\left(-\theta_{l, i}-\beta_{l, j}+\alpha / 4\right), b_{l, i j}=\left(-\theta_{l, i}+\beta_{l, j}+\alpha / 4\right), c_{l, i j}=\left(\theta_{l, i}-\beta_{l, j}+\alpha / 4\right)$, $d_{l, i j}=\left(\theta_{l, i}+\beta_{l, j}+\alpha / 4\right)$ with $\theta_{l, i}=0.5 \mu_{l} /(\Delta x)_{i+\frac{1}{2}}$ and $\beta_{l, j}=0.5 \eta_{l} /(\Delta y)_{j+\frac{1}{2}}$. The system (5.4) is explicitly solved using the sweeping method. 
Stability result. Let $\alpha$ be a positive constant. We have $\left\|(A+\alpha I)^{-1}\right\| \leq \frac{1}{\alpha}$ and the solution $f$ of equation (4.4) verifies $\|f\| \leq\|\varphi\| / \alpha$. It follows using piecewise bilinear approximation in space and piecewise constant approximation in angle, that the solution $\left\{f_{l, i+1 / 2 j+1 / 2}\right\}$ of (5.3) satisfies

$$
\left|f_{h}\right|_{h} \leq \frac{1}{\alpha}\left|\varphi_{h}\right|_{h}
$$

where $|\cdot|_{h}$ is the following discrete analogue of the norm $\|\cdot\|$ :

$$
\left|f_{h}\right|_{h}^{2}=\sum_{i=0}^{N-1} \sum_{j=0}^{M-1} \sum_{l=1}^{J}(\Delta x)_{i+\frac{1}{2}}(\Delta y)_{i+\frac{1}{2}} W_{l} f_{l, i+1 / 2 j+1 / 2}^{2}
$$

with $W_{l}$ being the weight associated to the angular node $\left(\mu_{l}, \eta_{l}\right) \in \Omega_{J}$. Thus the discrete scheme (5.3) is stable. Moreover this scheme is of the second order of accuracy and it is non monotonic. The monotonicity of the scheme can be achieved in the limit case where $h \rightarrow 0$ [9].

\subsection{Numerical Results}

We took particular data for which an exact solution of problem (2.1) is known in each case. For the iterative methods tested here, the iterations are stopped when the relative error $\left\|U-U_{\text {exate }}\right\|_{2} /\left\|U_{\text {exate }}\right\|_{2}$ is less than a prescribed $\varepsilon>0$.

Let $N_{s}$ and $N_{a}$ denote the total number of spatial grid points an discrete angular directions respectively. At each iteration of the SAS method, we have the following computational cost in one and two dimensional space isotropic cases:

- The cost of the sweeping algorithm is $O\left(N_{s} N_{a}\right)$ floating point operations (specifically $4 N_{s} N_{a}$ for $1-\mathrm{D}$ and $8 N_{s} N_{a}$ for $2-\mathrm{D}$ ).

- The solution of equation (4.2) using (3.7) needs $O\left(N_{s} N_{a}\right)$ floating point operations.

- Updating the right hand side took $O\left(N_{s} N_{a}\right)$ floating point operations. The overall computational cost at each iteration is $O\left(N_{s} N_{a}\right)$.

In the anisotropic case, the computational cost of each iteration is given by $O\left(n_{C G} N_{s} N_{a}^{2}\right)$, where $n_{C G}$ is the number of iterations necessary for the convergence of $\mathrm{CG}$ algorithm. It decreases with the number of SAS iteration. In both cases, there is no need in matrix storage.

Slab geometry case. Let take $a=0, b=1$. For $x \in(0,1)$ and $\mu, \mu^{\prime} \in[-1,1]$ we set $\sigma(x)=\sigma, \kappa\left(x, \mu, \mu^{\prime}\right)=\sigma c / 2$ and

$$
q(x, \mu)= \begin{cases}\mu^{2}+\sigma \mu x-\sigma c / 4, & \mu>0, \\ \mu^{2}+\sigma \mu(x-1)-\sigma c / 4, & \mu<0 .\end{cases}
$$

The exact solution of this problem is given by:

$$
\psi(x, \mu)= \begin{cases}\mu x, & \mu>0 \\ \mu(x-1), & \mu<0\end{cases}
$$


In the numerical tests we take $N=10$ and $J=10$. We study the behaviour of the SAS method with respect to parameters $\alpha, c$ and $\sigma$. The theoretical number of SAS iterations is minimal for $\alpha_{t}=\sigma(1-c)$. The SAS algorithm was applied for several values of $c$ and $\sigma$. For fixed $c$ and $\sigma$, the numerical optimal value of $\alpha$ can be localized in the interval $[\sigma(1-c), \sigma(1-c / 2)]$. We take

$$
\alpha= \begin{cases}\sigma(1-19 c / 32), & 0<c<0.9 \\ \sigma(1-23 c / 32), & 0.9 \leq c<0.97 \\ 1, & 0.97 \leq c \leq 1\end{cases}
$$

We compare the number of iterations and the CPU time of the standard Source Iteration (SI) method and the SAS method. There are two sets of tests: one for a fixed value of $c$, another for a fixed $\sigma$.
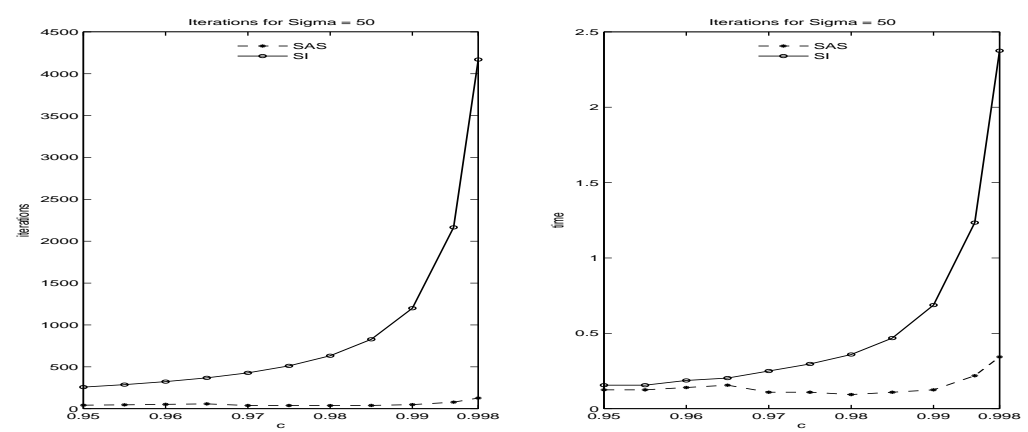

Figure 1. Comparison at fixed $\sigma=50$ of Source Iteration and SAS in slab geometry for values of $c$ close to 1 ( $\epsilon=1 E-06$ ): (left) Number of iterations; (right) CPU time.

As shown in Figure 1 - Figure 3, the SAS method converges faster than the standard SI method, particularly for large values of $\sigma$ and $c$ close to 1 . For $c=0.99$, we compare the $\sigma$ dependence of the iterative methods used here. We can see on Figure 2 that the number of iterations of the SAS method is the same as that of the ISAS method using the Conjugate Gradient (ISAS(CG)) method for the first inner equation. It can also be seen that the computing times of SAS and ISAS(CG) are roughly the same and are less than that of the SI method. This observation remains true for very large values of $\sigma$ (see, Figure 3).

Two dimensional case. Let $D=(0,1) \times(0,1)$. For $x=\left(x_{1}, x_{2}\right) \in D$ and $\Omega=(\mu, \eta) \in B(0,1)=\left\{\Omega \in \mathbb{R}^{2},\|\Omega\|_{2}<1\right\}$, we set $\sigma(x)=\sigma, \kappa\left(x, \Omega, \Omega^{\prime}\right)=\frac{\sigma c}{\pi}$,

$$
q(x, \mu)=\left\{\begin{array}{l}
\mu x_{2}+\eta x_{1}+\sigma x_{1} x_{2}-\sigma c / 4, \quad \mu>0, \quad \eta>0, \\
-\mu x_{2}+\eta\left(1-x_{1}\right)+\sigma\left(1-x_{1}\right) x_{2}-\sigma c / 4, \quad \mu<0, \quad \eta>0 \\
-\mu\left(1-x_{2}\right)-\eta\left(1-x_{1}\right)+\sigma\left(1-x_{1}\right)\left(1-x_{2}\right)-\sigma c / 4, \quad \mu, \eta<0 \\
\mu\left(1-x_{2}\right)-\eta x_{1}+\sigma x_{1}\left(1-x_{2}\right)-\sigma c / 4, \quad \mu>0, \quad \eta<0
\end{array}\right.
$$



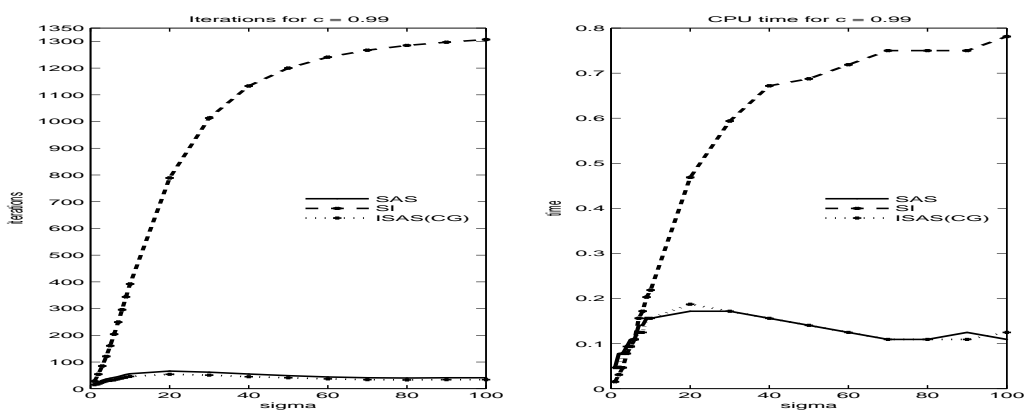

Figure 2. Comparison at fixed $c=0.99$ of Source Iteration, SAS and ISAS(CG) in slab geometry $(\epsilon=1 E-06)$ : (left) Number of iterations; (right) CPU time.
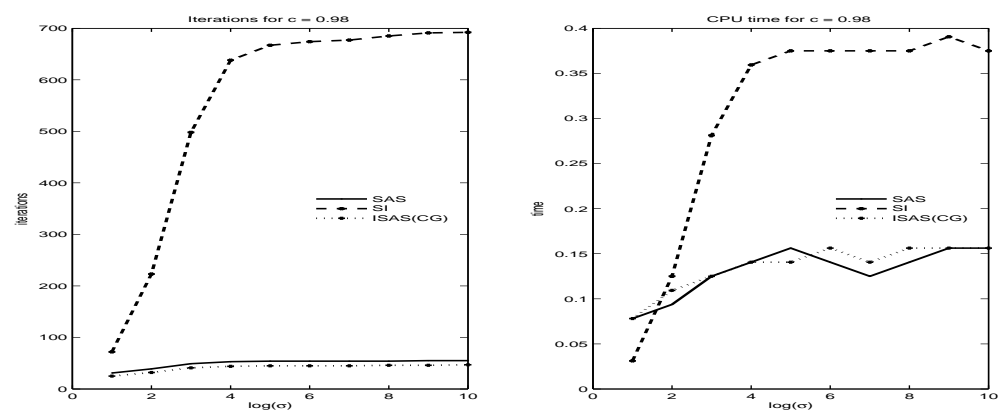

Figure 3. Comparison at fixed $c=0.98$ of Source Iteration, SAS and ISAS(CG) in slab geometry for large values of $\sigma(\epsilon=1 E-06)$ : (left) Number of iterations; (right) CPU time.

The exact solution of this test problem is given by:

$$
\psi(x, \mu)=\left\{\begin{array}{l}
x_{1} x_{2}, \quad \mu>0, \quad \eta>0, \quad\left(1-x_{1}\right) x_{2}, \quad \mu<0, \quad \eta>0 \\
\left(1-x_{1}\right)\left(1-x_{2}\right), \quad \mu<0, \quad \eta<0, \quad x_{1}\left(1-x_{2}\right), \quad \mu>0, \quad \eta<0 .
\end{array}\right.
$$

For the numerical tests, we take $\Delta x=\Delta y=\frac{1}{10}$ and $J=100$. We perform the same set of tests as in the case of slab geometry, using the previous values of $\alpha$ for SAS iteration. For $c=0.98$, we compare the $\sigma$ dependence of the methods tested here. It can be seen (see, Figure 4) that SAS method is more efficient than the SI method, even for large values of $\sigma$ (see, Figure 5).

We also compare the $c$ dependence of the two iteration methods for $\sigma=50$, with $c$ near to 1 (see, Figure 6). We can see that the SAS algorithm is still more efficient. We plot in Figure 7 the convergence rate of the two methods at $c=0.5$ and $c=1$ for several values of $\sigma$. We can see that SAS algorithm converges for $c=1$, even for very large values of $\sigma$. As in the case of slab geometry, the SAS algorithm is more efficient than the SI algorithm, particularly for the critical cases ( $c$ close to 1 and/or large $\sigma$ ). 

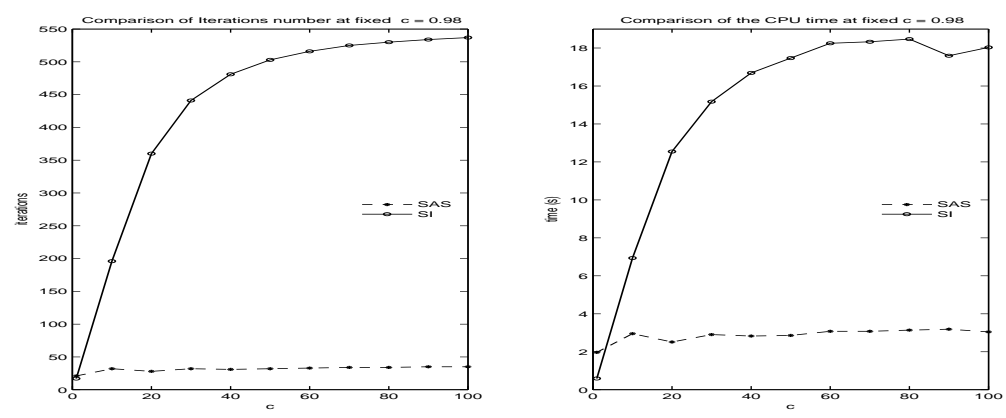

Figure 4. Comparison of the SI and SAS methods in 2D case at fixed $c=0.98(\epsilon=1 E-5)$ : (left) number of iterations; (right) CPU time.
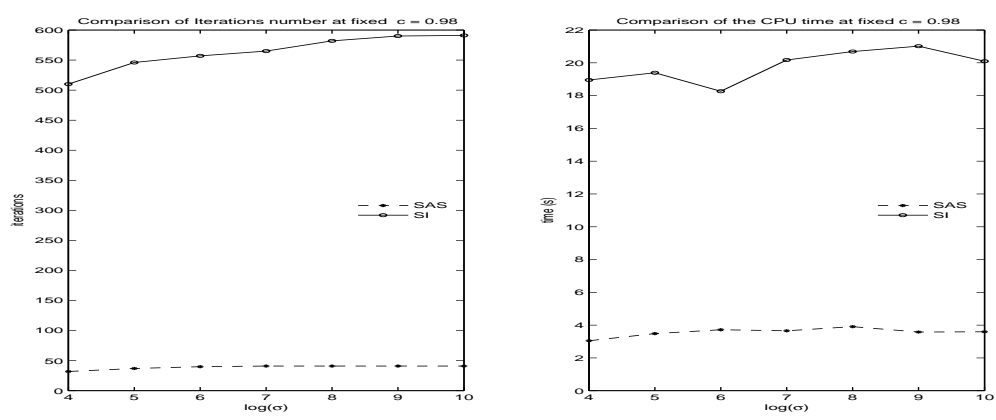

Figure 5. Comparison of the SI and SAS methods in $2 \mathrm{D}$ case at fixed $c=0.98$, for large $\sigma$ $(\epsilon=1 E-5)$ : (left) number of iterations; (right) CPU time.

Additionally, we present in Table 1 comparative numerical results (number of iteration and CPU time) of SAS and $\operatorname{IDR}(s)$ ( $s$ denoting the dimension of the Krylov subspace), SSOR preconditioned GMRES (fgmres), BiCGStab iterative algorithm with Gauss-Seidel preconditioner (Bicgstab) and a spatial multigrid method (MG). For this set of tests, we take $\Delta x=\Delta y=\frac{1}{8}$ and $J=100$. The matrix $G$ of the resulting discrete system has 2562500 non zeros entries. The iterations are stopped when the relative error $\|B-G U\|_{2} /\|B\|_{2}$ is less than $1 E-05$, where $B$ denotes the right hand side of the discrete system. The convergence behaviour (relative residual as function of iteration) of the SAS,

Table 1. Iterations number and CPU time in s (in bracket) at $\sigma=100$.

\begin{tabular}{rcccccc}
\hline & MG & SAS & IDR(4) & IDR(16) & Bicgstab & fgmres \\
\hline$c=0.5$ & $23(107.87)$ & $7(0.95)$ & $87(5.28)$ & $82(2.29)$ & $41(11.28)$ & $32(5.01)$ \\
$c=0.98$ & $>200$ & $33(4.32)$ & $125(7.56)$ & $106(7.15)$ & $77(20.97)$ & $95(15.34)$ \\
\hline
\end{tabular}



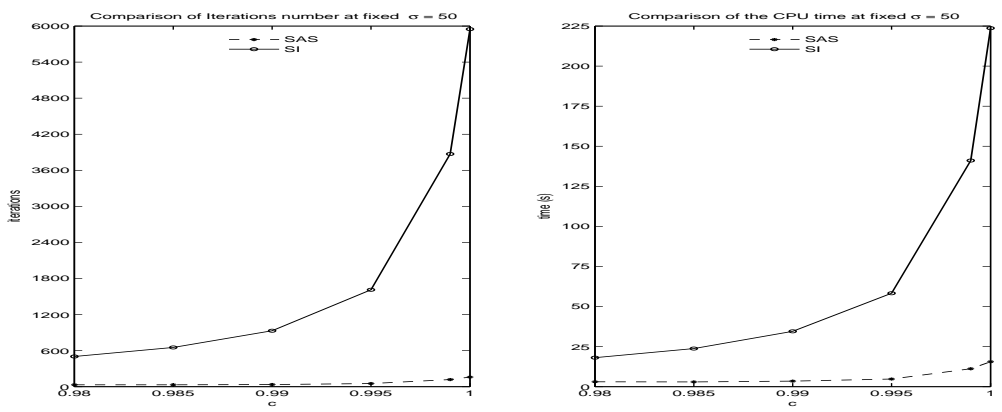

Figure 6. Comparison of the SI and SAS methods in $2 \mathrm{D}$ case at fixed $\sigma=50$, for $c \approx 1$ $(\epsilon=1 E-05)$ : (left) number of iterations; (right) CPU time.
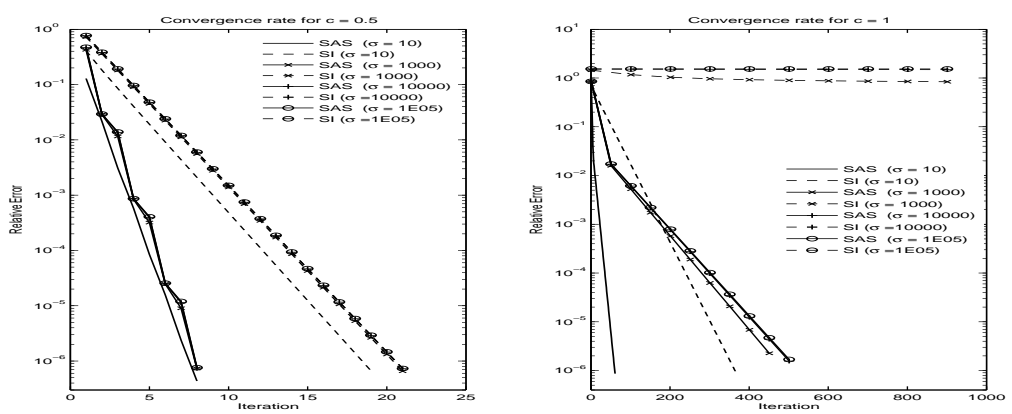

Figure 7. Comparison of the convergence rate in $2 \mathrm{D}$ case at fixed $c$ of SI and SAS iteration for several values of $\sigma$ : (left) $c=0.5$ and (right) $c=1$.

MG and FGMRES are plotted in Figure 8 and Figure 9 for the cases $c=0.5$ and $c=0.98$ respectively. It can be observed that the SAS method is efficient in both cases as compared to the other methods tested here.

\section{Conclusion}

Throughout this work, it comes that the iterative methods based on a Selfadjoint and $m$-Accretive splitting of the transport operator presented, converge unconditionally to the solution of the transport equation. The previous numerical results show the feasibility and the effectiveness of the SAS iteration. It appears that the SAS iteration is efficient compared to the standard Source Iteration. The method converges for critical cases ( $c$ close to 1 and/or large $\sigma)$ and it can be easily implemented in $1 \mathrm{D}$ and $2 \mathrm{D}$ dimensions. It is important to mention that the theoretical proof of the convergence of the method is independent of the discretization. 

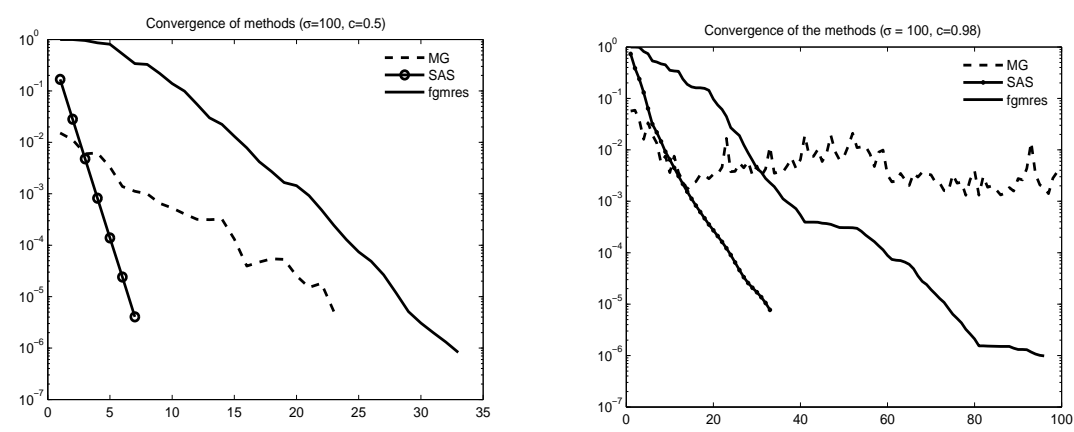

Figure 8. Convergence of the SAS, fgm- Figure 9. Convergence of the SAS, fgmres res and MG $(c=0.5)$ methods at fixed and MG $(c=0.98)$ methods at fixed $\sigma=$ $\sigma=100$. 100.

\section{Acknowledgment}

The authors wish to express their gratitude to anonymous referees for their useful remarks and comments which improved the presentation of this paper.

\section{References}

[1] S. Akesbi and E. Maître. Minimal residual method applied to the transport equation. Numerical Algorithms, 26(3):235-249, 2001.

[2] S. Akesbi and E. Maître. Theoretical and numerical analysis of a minimal residual solver for 2D Boltzmann equation. Journal of Computation and Applied Mathematics, 150(2):357-374, 2003.

[3] R. Barrrett, M. Berry, T.F. Chan, J. Demmel, J. Donato, J. Dongarra, V. Eijkhout, R. Pozo, C. Romine and H. Van der Vorst. Templates for the Solution of Linear Systems: Building Blocks for Iterative Methods. SIAM, Philadelphia, 1994.

[4] V. Brattka and R. Dillhage. Computability of the spectrum of self-adjoint operators. Journal of Universal Computer Science, 11:1884-1900, 2005.

[5] H. Brezis. Analyse Fonctionnelle. Theorie et Application. Masson, Paris, 1987. (2 eme tirage)

[6] B. Chang and B. Lee. A multigrid algorithm for solving the multi-group anisotropic scattering Boltzmann equation using first-order system least-squares methodology. Electronic Transactions on Numerical Analysis, 15:132-151, 2003.

[7] R. Čiegis, O. Iliev and Z. Lakdawala. On parallel numerical algorithms for simulating industrial filtration problems. Computational Methods in Applied Mathematics, 7(2):118-134, 2007.

[8] Raim. Čiegis, Rem. Čiegis, M. Meilūnas, G. Jankevičiūtè and V. Starikovičius. Parallel numerical algorithm for optimization of electrical cables. Math. Model. Anal., 13(4):471-482, 2008. (doi:10.3846/1392-6292.2008.13.471-482) 
[9] R. Dautray and J.-L. Lions. Analyse Mathématique et Calcul Numérique pour les Sciences et les Techniques, Tome 3. Masson, Paris, 1985.

[10] T. A. Manteuffel, S. McCormick, J.E. Morel and G. Yang. A fast multigrid algorithm for isotropic transport problems ii. with absorption. SIAM Journal on Scientific Computing, 17:1449-1474, 1996.

[11] T. A. Manteuffel and K. Ressel. Least-squares finite element solution for the neutronic transport equation in diffusive regimes. SIAM J. Numer. Anal., 35, 1998.

[12] G. Marchuk. Decomposition Methods. Nauka, Moscow, 1988. (in Russian)

[13] G. Marchuk and V. Agochkov. Introduction aux Méthodes des Eléments Finis. Mir, Moscow, 1985.

[14] S. Oliveira and Y. Deng. Preconditionrd Krylov subspace methods for transport equations. Prog. Nucl. Energy, 33(1/2):155-174, 1998.

[15] B. W. Patton and J. P. Holloway. Preconditioned GMRES to the numerical solution of the neutron transport equation. Ann. Nucl. Energy, 29(2), 2002.

[16] P. Sonneveld and M. B. van Gijzen. IDR(s): a family of simple and fast algorithms for solving large nonsymmetric systems of linear equations. Preprint, Delft Institute of Applied mathematics, Delft University of Technology, Delft, The Netherlands, March 2007.

[17] F. D. Swesty, D. C. Smolarski and P. E. Saylor. A comparison of algorithms for the efficient solution of the linear systems arising from multigroup flux-limited diffusion problems. The Astrophysical Journal Supplement Series, 182:369-387, 2004 .

[18] A. Tizaoui. Splitting operator for solving the neutron transport equation in 1-D spherical geometry. International Journal of Mathematics and Statistics, 1(A07):31-45, 2007.

[19] J. S. Warsa, M. Benzi, T. A. Wareing and J. E. Morel. Fully consistent diffusion synthetic acceleration of linear discontinuous transport discretizations on threedimensional unstructured meshes. Nucl. Sci. Engr., 141:236-251, 2002.

[20] J. S. Warsa, M. Benzi, T. A. Wareing and J. E. Morel. Preconditioning a mixed discontinuous finite element method for radiation diffusion. Linear Algebra Appl., 11:795-811, 2004. 